\title{
Marker Cluster Rigidity in a Multi-Segment Foot Model
}

\author{
Po-Hsiang Chan ${ }^{\mathrm{a}}$, Julie Stebbins ${ }^{\mathrm{b}}$, Amy B. Zavatsky ${ }^{\mathrm{a}}$ \\ ${ }^{a}$ Department of Engineering Science, University of Oxford, Oxford, UK \\ ${ }^{\mathrm{b}}$ Oxford Gait Laboratory, Nuffield Orthopaedic Centre NHS Trust, Oxford, UK
}

Corresponding author: Prof Amy B. Zavatsky

Address: Department of Engineering Science, University of Oxford, Parks Road, Oxford OX1 3PJ, UK

Email: amy.zavatsky@eng.ox.ac.uk

Keywords: Rigidity; Foot Model; Forefoot; Gait Analysis; Marker Cluster

Accepted for publication in Journal of Biomechanics

1 January 2019

Page: 1 


\begin{abstract}
Multi-segment foot models (MSFM) are used in gait analysis for the diagnosis and planning of treatment for patients with foot deformities. Like other biomechanical models, MSFMs represent the leg and foot as a series of linked rigid segments, but such a simplification may not be appropriate, particularly for the flexible forefoot. This study investigated the appropriateness of the rigid body assumption on marker clusters used to define the individual segments (tibia, hindfoot, forefoot) of a widely-used MSFM. Rigidity of the marker clusters was quantified using the rigid body error $\left(\sigma_{R B E}\right)$ calculated for each frame of a representative gait cycle for 64 normal healthy adults who underwent gait analysis. $\sigma_{R B E}$ is a measure of how well the tracking marker configuration at each frame compares to the arrangement of the same markers in a reference pose. As expected, the patterns of deformation of the three marker clusters differed over the gait cycle. The hindfoot cluster remained relatively undeformed in comparison to the forefoot and tibia clusters. The largest deformations of the forefoot cluster occurred near the beginning and end of the stance phase. The tibia cluster deformed throughout the entire gait cycle, with a pattern similar to that of a typical knee flexion angle graph. On average, the forefoot cluster was the least rigid, followed by the tibia cluster and then the hindfoot cluster. The results raise questions about the appropriateness of the rigid-body assumption when applied to MSFMs, particularly in the forefoot region.
\end{abstract}




\section{Introduction}

Multi-segment foot models (MSFM) have become increasingly popular due to the improved accuracy and ease-of-use of motion capture technology, and there is strong evidence supporting their clinical use (Wren et al., 2011). Sub-division of the foot into multiple segments for gait analysis has helped with the diagnosis and planning of treatment for foot deformities, as well as furthering our understanding of foot biomechanics. There are many different MSFMs used in clinical gait laboratories worldwide, including the Oxford Foot Model (OFM) (Carson et al., 2001; Stebbins et al., 2006), the Rizzoli Foot Model (Leardini, Benedetti et al., 2007), the Heidelberg Foot Measurement Method (Simon et al., 2006), and several others (Deschamps et al., 2011; Rankine et al., 2008). These all share some key features, such as a hindfoot segment, but differ in others, notably in how the bones in the rest of the foot are modelled.

Although most lower-limb biomechanical models used in gait analysis represent the leg and foot as a series of linked rigid segments, such a simplification may not be appropriate in a MSFM, even if it makes the related measurements and calculations easier. Whether the midfoot and forefoot in particular should be modelled as rigid bodies is questionable, given the known flexibility of the medial longitudinal arch and the mediolateral spread of the forefoot during the stance phase of gait (Duerinck et al., 2014).

In classical mechanics, a rigid body is characterized by the requirement that the distance between any two points on the body remains fixed. Therefore, the simplest way to quantify deformation is to find the change in Euclidean distance between the points or, alternatively, to calculate the strain. An alternative measure of deformation which uses

multiple points at once is the rigid body error $\left(\sigma_{R B E}\right)$ introduced by van den Bogert et al. (1994) for the purpose of quantifying soft-tissue artefact. The $\sigma_{R B E}$ effectively quantifies violations of the rigid-body assumption. In the present context, it can be thought of as a 
measure of the difference between the configuration of selected points (identified by clusters of skin-mounted markers) on the surface of a body segment in a reference pose and the configuration of those same points (or markers) after the body has changed position.

This study aimed to quantify the $\sigma_{R B E}$ of skin-mounted marker clusters on the forefoot, hindfoot, and tibia ${ }^{1}$ segments of a multi-segment foot model widely used in clinical gait analysis (Carson et al., 2001; Stebbins et al., 2006), and, by doing so, to gain some understanding of their apparent "rigidity" and the appropriateness of a rigid-body assumption. For normal healthy adult subjects, it was hypothesized that on average the forefoot cluster would be less rigid than the clusters on the other two segments and that the deformation patterns of the clusters would differ over the gait cycle.

\section{Methods}

\subsection{Data collection}

Sixty-four healthy adult subjects (32 male, 32 female) with an average age of 27.3 \pm 7.0 years participated in the study, which was approved by the local Ethics Committee. All participants gave informed consent. Retroreflective markers were placed on the lower limbs as for the OFM (Stebbins et al., 2006) (Figure 1). Subjects first posed in a static standing position and then walked along a 10-m walkway at a self-selected speed while lower-limb motion data (Vicon Motion Systems Ltd., Oxford, UK) was collected at $100 \mathrm{~Hz}$ and ground reaction force data (Advanced Mechanical Technology, Inc., Watertown, USA) collected at $1000 \mathrm{~Hz}$. Marker gap filling, trajectory filtering, and computation of OFM joint angles were conducted using Vicon Nexus (v2.3). One of six walking trials (right leg only) was selected for further analysis based on how close its OFM joint angle graphs were to the average OFM joint angle graphs for that subject. The quantity used for the comparisons was the root mean

\footnotetext{
${ }^{1}$ Note that, in some models, this segment is referred to as the "shank" since it comprises both the tibia and the fibula.
} 
square error. For quality assurance, the temporal-spatial parameters and joint angles were inspected to ensure that there were no abnormal features or measurement artefacts.

\subsection{Quantification of marker cluster rigidity}

Rigidity was quantified using the rigid body error $\sigma_{R B E}$ (Söderkvist and Wedin, 1993; van den Bogert et al., 1994), calculated as:

$$
\sigma_{R B E}=\sqrt{\frac{1}{3 N-6}}\left\|\left[T^{*}\right][A]-[B]\right\|
$$

where $N$ is the number of markers, $[A]$ the reference configuration of the markers (as given by their Cartesian coordinates in a global reference frame), $[B]$ any other configuration of the markers (again given by their spatial coordinates), and $\left[T^{*}\right]$ the orthogonal transformation matrix such that the expression $\left\|\left[T^{*}\right][A]-[B]\right\|$ is minimised. The minimisation (orthogonal Procrustes) problem was solved using the method proposed by Söderkvist and Wedin (1993). This algorithm finds the best-fit rigid-body transformation from a reference pose to any other body position, which implies that the quantity given by $\left(\left\|\left[T^{*}\right][A]-[B]\right\|\right)$ indicates how much the body or, in the present case, the marker cluster defining it, has deformed.

The $\sigma_{R B E}$ for marker clusters on the OFM tibia, hindfoot, and forefoot segments (Figure 1) was calculated over one full gait cycle using MATLAB (v2015a, MathWorks Inc., Natick, USA). Two reference poses for the calculations were used: the marker cluster configuration in the static standing position and that at mid-stance (50\% stance phase). The average rigid body error $\left(\sigma_{a v}\right)$ was used to indicate the average rigidity of each marker cluster, and the peak-to-peak rigidity $\left(\sigma_{p t p}\right)$ characterized its maximum deformation. 


\subsection{Statistics}

The repeatability of the measurement protocol and the variability of $\sigma_{R B E}$ were assessed as described in Appendix A. For the comparison of the $\sigma_{R B E}$ between segments (for the mid-stance reference pose only), the data were analyzed using a two-way repeated measures ANOVA with gender (male or female) as the between-subject factor and body segment (forefoot, hindfoot, and tibia) as the within-subject factor. The motivation behind this investigation was that there are known anthropometric differences between the male and female foot, which influence the dynamic shape deformation (Fritz et al., 2013) throughout gait.

Mauchley's test was conducted to inspect the homogeneity of variance across withinsubject factors. If significant, the Greenhouse-Geisser corrected p-value was reported, otherwise, the uncorrected $p$-value was used. If significant interactions $(p<0.05)$ were found between factors, post-hoc comparisons were performed using an unpaired t-test with Bonferroni corrections. All statistical calculations were completed using SPSS (v24, IBM Corp., Armonk, USA).

\section{Results}

As hypothesized, the patterns of deformation of the marker clusters differed over the gait cycle (Figures 2 and 3). Use of the static standing position for the reference marker cluster configuration resulted in a more variable $\sigma_{R B E}$, as indicated by the standard deviations in Tables 1 and 2. Regardless of reference position, the hindfoot cluster stayed relatively undeformed in comparison to the forefoot and tibia clusters. For the forefoot cluster, the largest deformations occurred near the beginning and end of the stance phase, as indicated by 
the sharp fall and rise in $\sigma_{R B E}$. As opposed to what was expected, the tibia cluster also deformed throughout the entire gait cycle.

There was no statistically significant interaction between the effects of gender and $\sigma_{a v}$ $(\mathrm{F}(2,62)=0.229, \mathrm{p}=0.80)$; however, there were statistically significant differences between the average rigidities of the three clusters $(\mathrm{p}<0.01)$ (Table 2). As hypothesized, the forefoot cluster was the least rigid. It had the largest $\sigma_{a v}$, followed by the tibia cluster and then the hindfoot cluster. The p-value was less than 0.01 in all pairwise comparisons between the three clusters. The hindfoot cluster exhibited the lowest $\sigma_{p t p}(\mathrm{p}<0.01)$. The tibia $\sigma_{p t p}$ was significantly lower than that of the forefoot cluster $(\mathrm{p}=0.01)$.

\section{Discussion}

Residuals from the marker tracking algorithm have been used to study the tracking error (Söderkvist and Wedin, 1994; Spoor and Veldpaus, 1980), to assess the soft tissue artefact (van den Bogert et al., 1994), and to help minimize the transformation matrix error (Leitch et al., 2010). In the present study, a different approach was taken, with the residual $\sigma_{R B E}$ used to quantify the rigidity (or deformation) of a cluster of markers used to track a segment. For normal healthy adults, it was confirmed that on average the forefoot cluster of a widely used MSFM was less rigid than the hindfoot cluster and that their patterns of deformation differed over the gait cycle. This was expected since all hindfoot markers were on one bone - the calcaneus, whereas the forefoot markers were spread across the metatarsals. Both skin-movement error and segment flexibility are captured in the $\sigma_{R B E}$ of the marker clusters, and although the latter is likely to be more dominant in the forefoot, this study is unable to verify how much each effect contributes to the total $\sigma_{R B E}$.

The deformations of the hindfoot and forefoot marker clusters are consistent with our general understanding of how foot shape changes during gait (Baker, 2013; Levine et al., 
2012). The forefoot markers most nearly resemble their mid-stance reference configurations during the single-limb support phase of stance (hence the lower $\sigma_{R B E}$ in this period); this is when the load is transmitted through the forefoot and the metatarsals are most spread. The forefoot deforms rapidly at two periods during the stance phase, the loading response and pre-swing. At loading response, the foot is lowered to the ground through plantarflexion of the ankle. It is during this period that the distance between the first and fifth metatarsals increases (Duerinck et al., 2014). During pre-swing, the forefoot returns to its unloaded configuration.

As opposed to what was expected, the tibia marker cluster deformed throughout the entire gait cycle, with the plot of its $\sigma_{R B E}$ resembling a characteristic knee flexion-extension angle graph, especially in Figure 2(e,f). The largest deformation occurred from mid-swing to terminal swing. Since the only potential source of bone motion in the OFM tibia segment is between the tibia and fibula, which are known to have very limited motion relative to each other (Brockett and Chapman, 2016; Soavi et al., 2000), any deformation was most likely due to skin or other soft tissue movements. Given the relatively large range of knee motion and the proximity of the marker on the fibula head to the knee joint, this marker is likely to be the one with excessive skin motion. This interpretation is consistent with the results of a fluoroscopic study of the soft tissue artefact of markers on the shank by Tsai et al. (2009).

The exact numerical values of $\sigma_{R B E}$ depend on choice of the tracking markers used in the calculations and on the chosen reference configurations of the markers (compare Figures 2 and 3). The choice of tracking markers was based on those used clinically in the MSFM, since straightforward application of the results to clinical work was deemed to be important. The effects of marker and configuration choices are likely to be larger than the repeatability errors, which here were an order of magnitude smaller than the $\sigma_{a v}$ values for each segment (compare Tables 1, 2, and A.1). Regardless of marker choice, marker placement consistency 
and experience are key, as is also true for the measurement of gait kinematics (Caravaggi et al., 2011; McCahill et al., 2018; McGinley et al., 2009).

The results raise questions about the appropriateness of the rigid-body assumption when applied to MSFMs, particularly in the forefoot region. This should prompt consideration of how best to model the foot. The information given by $\sigma_{R B E}$ could be usefully exploited, for instance, in comparing objectively different marker clusters or sub-divisions of the foot in MSFMs to identify the ones that maintain the highest rigidity during activities. It should also stimulate the development of new ways in which to model the natural flexibility of the foot.

\section{Conclusion}

This study showed that $\sigma_{R B E}$ can be used successfully to quantify the overall rigidity of marker clusters representing the tibia, hindfoot, and forefoot segments of a MSFM, as well as to monitor how each cluster deforms throughout the gait cycle. Overall, the forefoot cluster was the least rigid, followed by the tibia cluster, and then the hindfoot cluster.

\section{Conflict of interest statement}

The authors declare that there are no conflicts of interest in the present study.

\section{Acknowledgements}

The authors would like to thank the team at the Oxford Gait Laboratory for their assistance and support in this project. 


\section{References}

Baker, R., 2013. Measuring Walking: A Handbook of Clinical Gait Analysis. Mac Keith Press.

Bartlett, J.W., Frost, C., 2008. Reliability, repeatability and reproducibility: Analysis of measurement errors in continuous variables. Ultrasound in Obstetrics and Gynecology. $31,466-475$.

Brockett, C.L., Chapman, G.J., 2016. Biomechanics of the ankle. Orthopaedics and Trauma $30,232-238$.

Caravaggi, P., Benedetti, M.G., Berti, L., Leardini, A., 2011. Repeatability of a multisegment foot protocol in adult subjects. Gait and Posture 33, 133-135.

Carson, M.C., Harrington, M.E., Thompson, N., O’Connor, J.J., Theologis, T.N., 2001. Kinematic analysis of a multi-segment foot model for research and clinical applications: a repeatability analysis. Journal of Biomechanics. 34, 1299-1307.

Deschamps, K., Staes, F., Roosen, P., Nobels, F., Desloovere, K., Bruyninckx, H., Matricali, G.A., 2011. Body of evidence supporting the clinical use of 3D multisegment foot models: A systematic review. Gait and Posture 33, 338-349.

Duerinck, S., Hagman, F., Jonkers, I., Van Roy, P., Vaes, P., 2014. Forefoot deformation during stance: Does the forefoot collapse during loading? Gait and Posture 39, 40-47.

Fritz, B., Schmeltzpfenning, T., Plank, C., Hein, T., Grau, S., 2013. Anthropometric influences on dynamic foot shape: Measurements of plantar three-dimensional foot deformation. Footwear Science 5, 121-129.

Leardini, A., Benedetti, M.G., Berti, L., Bettinelli, D., Nativo, R., Giannini, S., 2007. Rearfoot, mid-foot and fore-foot motion during the stance phase of gait. Gait and Posture 25, $453-462$.

Leitch, J., Stebbins, J., Zavatsky, A.B., 2010. Subject-specific axes of the ankle joint 
complex. Journal of Biomechanics 43, 2923-2928.

Levine, D., Ricahrds, J., Whittle, M.W., 2012. Whittle’s Gait Analysis. Churchill Livingstone.

McCahill, J., Stebbins, J., Koning, B., Harlaar, J., Theologis, T., 2018. Repeatability of the Oxford Foot Model in children with foot deformity. Gait and Posture 61, 86-89.

McGinley, J.L., Baker, R., Wolfe, R., Morris, M.E., 2009. The reliability of threedimensional kinematic gait measurements: A systematic review. Gait and Posture 29, $360-369$.

Rankine, L., Long, J., Canseco, K., Harris, G.F., 2008. Multisegmental foot modeling: a review. Critical Reviews in Biomedical Engineering 36, 127-181.

Schwartz, M.H., Trost, J.P., Wervey, R.A., 2004. Measurement and management of errors in quantitative gait data. Gait and Posture 20, 196-203.

Simon, J., Doederlein, L., McIntosh, A.S., Metaxiotis, D., Bock, H.G., Wolf, S.I., 2006. The Heidelberg foot measurement method: Development, description and assessment. Gait and Posture 23, 411-424.

Soavi, R., Girolami, M., Loreti, I., Bragonzoni, L., Monti, C., Visani, A., Marcacci, M., 2000. The mobility of the proximal tibio-fibular joint. A Roentgen Stereophotogrammetric Analysis on six cadaver specimens. Foot \& Ankle International 21, 336-342.

Söderkvist, I., Wedin, P.Å., 1994. On condition numbers and algorithms for determining a rigid body movement. BIT Numerical Mathematics 34, 424-436.

Söderkvist, I., Wedin, P.̊., 1993. Determining the movements of the skeleton using wellconfigured markers. Journal of Biomechanics 26, 1473-1477.

Spoor, C.W., Veldpaus, F.E., 1980. Rigid body motion calculated from spatial co-ordinates of markers. Journal of Biomechanics 13, 391-393.

Stebbins, J., Harrington, M., Thompson, N., Zavatsky, A., Theologis, T., 2006. Repeatability 
of a model for measuring multi-segment foot kinematics in children. Gait and Posture $23,401-410$.

Tsai, T.-Y., Lu, T.-W., Kuo, M.-Y., Hsu, H.-C., 2009. Quantification of three-dimensional movement of skin markers relative to the underlying bones during functional activities. Biomedical Engineering: Applications, Basis and Communications 21, 223-232.

van den Bogert, A.J., Smith, G.D., Nigg, B.M., 1994. In vivo determination of the anatomical axes of the ankle joint complex: An optimization approach. Journal of Biomechanics 27, $1477-1488$.

Wren, T.A.L., Gorton, G.E., Õunpuu, S., Tucker, C.A., 2011. Efficacy of clinical gait nalysis: A systematic review. Gait and Posture 34, 149-153. 

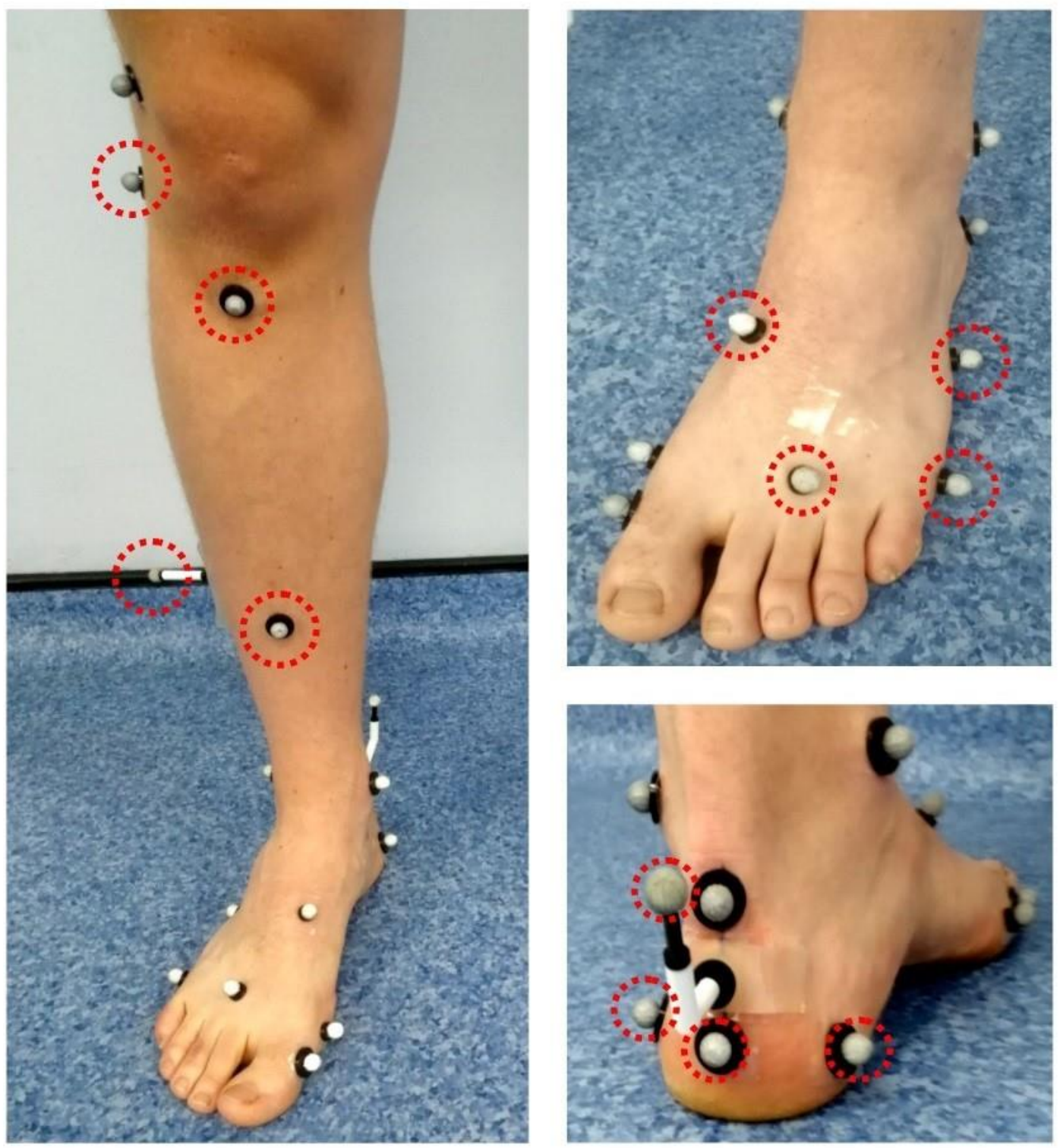

Figure 1: The OFM marker set (Carson et al., 2001; Stebbins et al., 2006). A cluster of four markers on each segment (shown circled in red) was used in the calculation of $\sigma_{R B E}$. On the hindfoot, the cluster markers were placed on the posterior distal aspect of the heel, sustentaculum tali, lateral calcaneus, and posterior calcaneus (wand marker). On the forefoot, the cluster markers were located between the second and third metatarsal heads, on the bases of first and fifth metatarsals, and on the head of the fifth metatarsal. The tibia cluster markers were placed on the head of the fibula, tibial tuberosity, anterior crest of the tibia, and the lateral surface of the tibia (wand marker). 
a) Forefoot (Male)

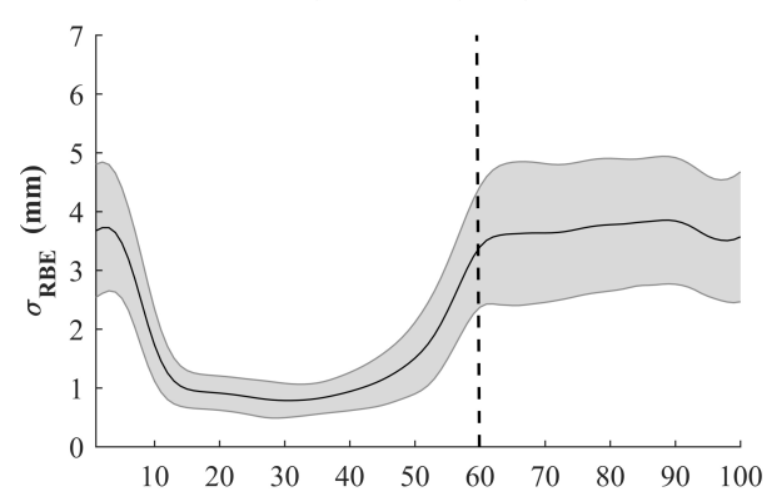

c) Hindfoot (Male)

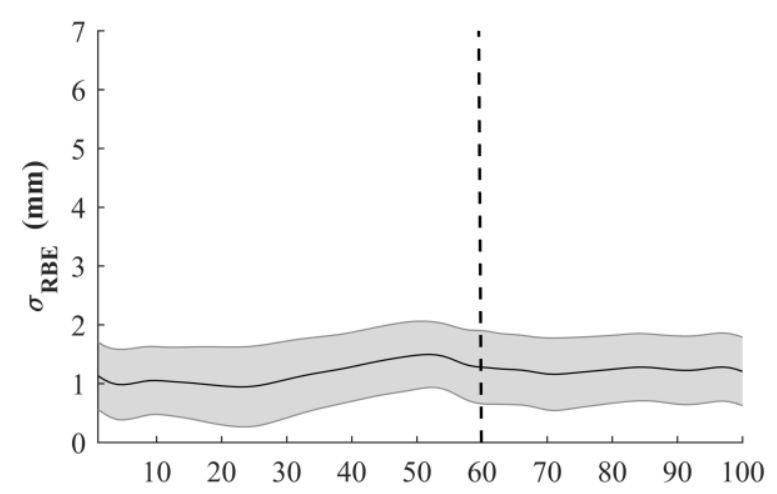

e) Tibia (Male)

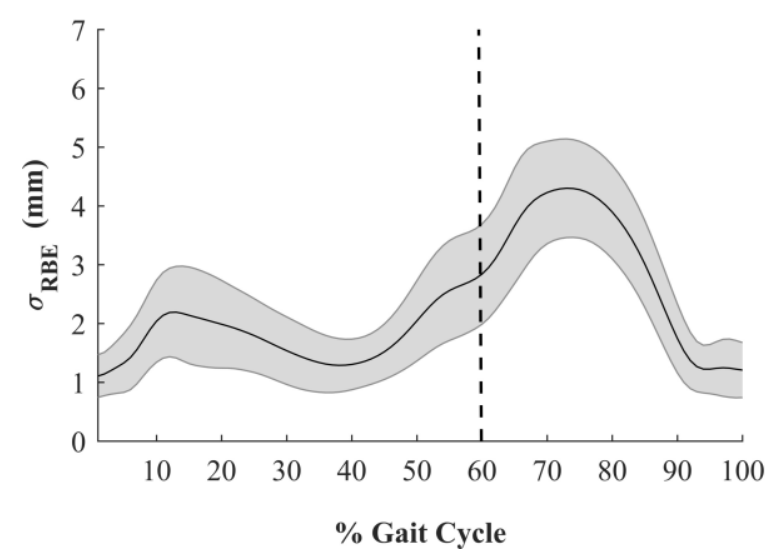

b) Forefoot (Female)

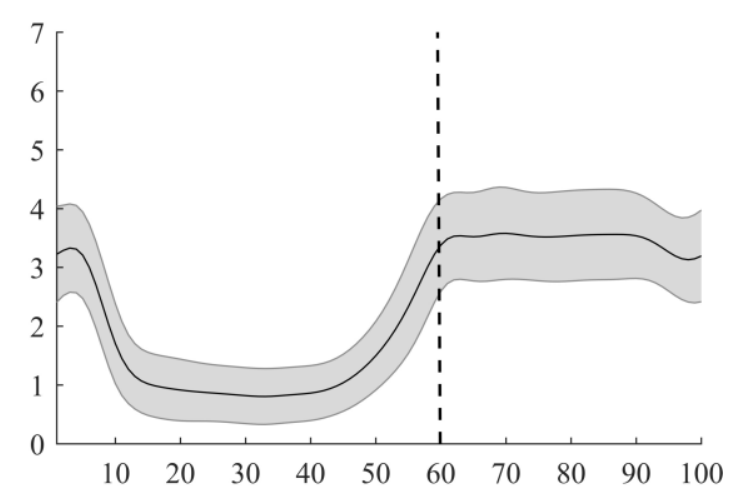

d) Hindfoot (Female)

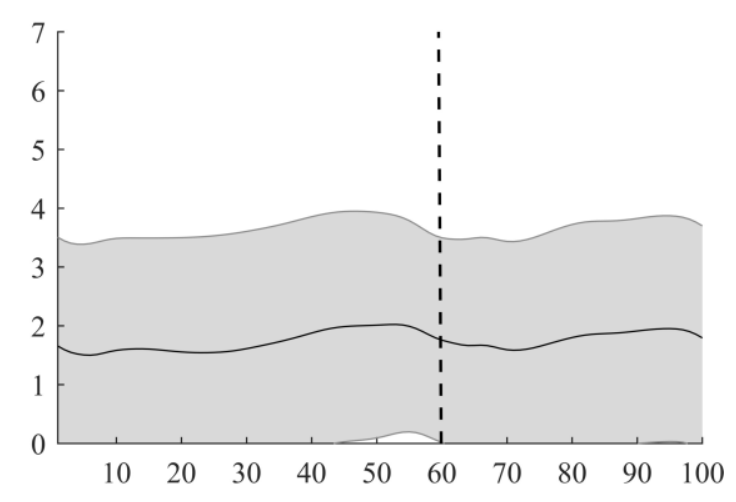

f) Tibia (Female)

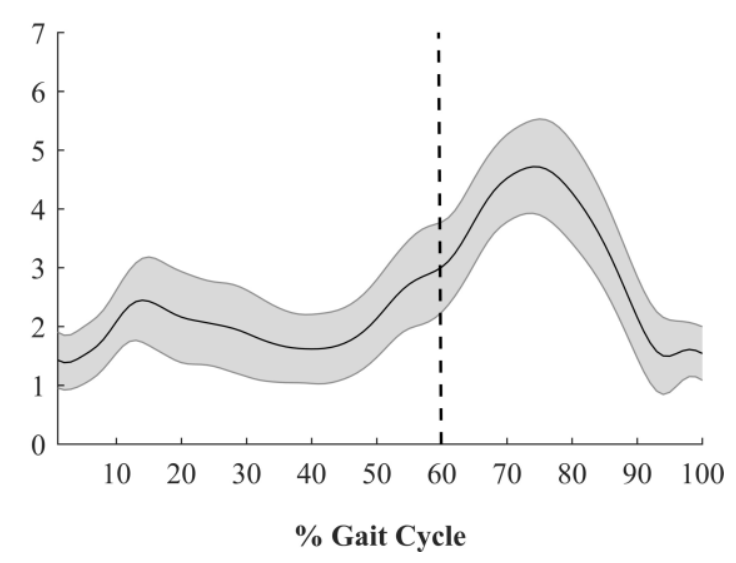

Figure 2: Forefoot, hindfoot, and tibia marker cluster rigid body error $\left(\sigma_{R B E}\right)$ over one full gait cycle calculated with the cluster configurations in the static trial as the reference. Means \pm 1 standard deviation $(\mathrm{mm})$. The dotted line indicates the separation between the stance phase and swing phase, with an average of $59.8 \%$ for the population tested in this study. Note that, on average, $\sigma_{R B E}$ is never equal to zero, since the marker cluster configurations during gait never match their configurations in the reference position. 
a) Forefoot (Male)

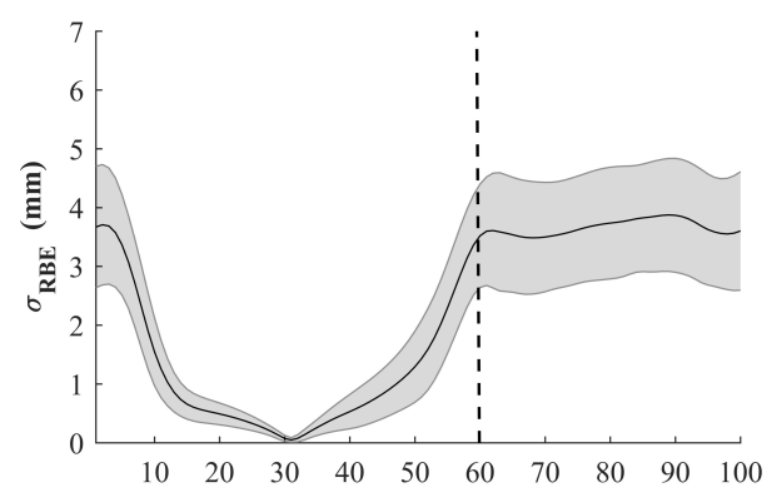

c) Hindfoot (Male)

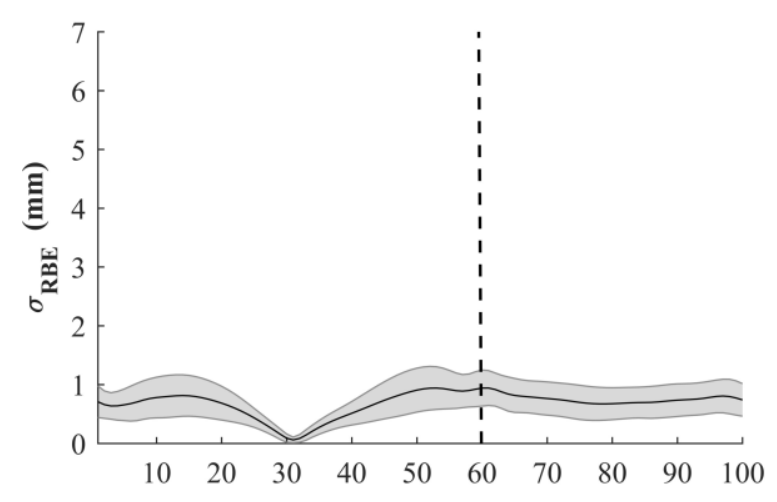

e) Tibia (Male)

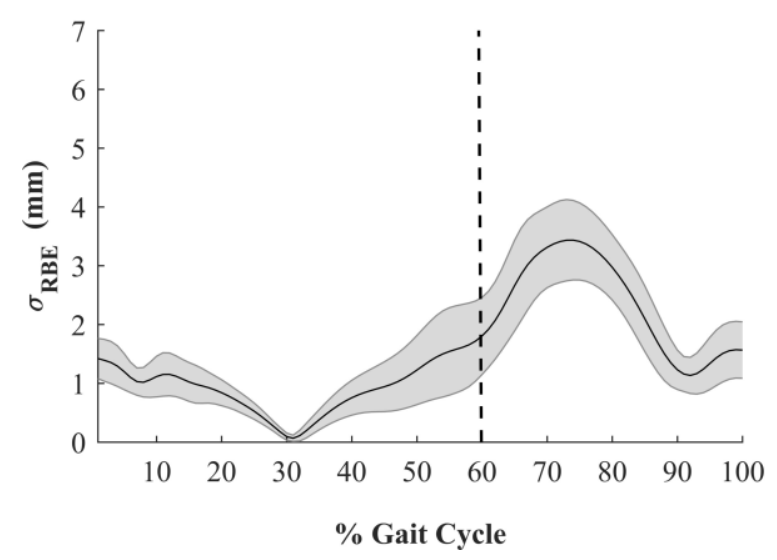

b) Forefoot (Female)

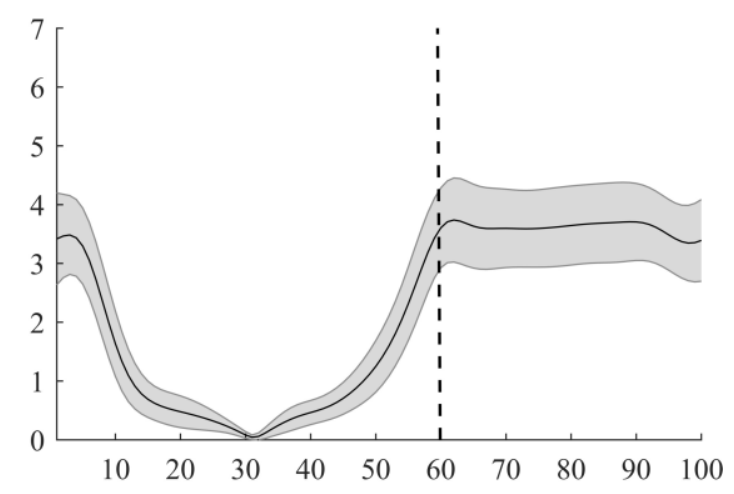

d) Hindfoot (Female)

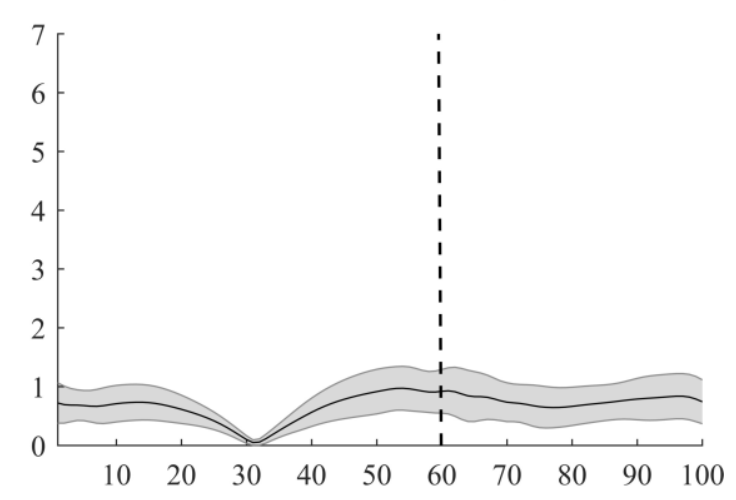

f) Tibia (Female)

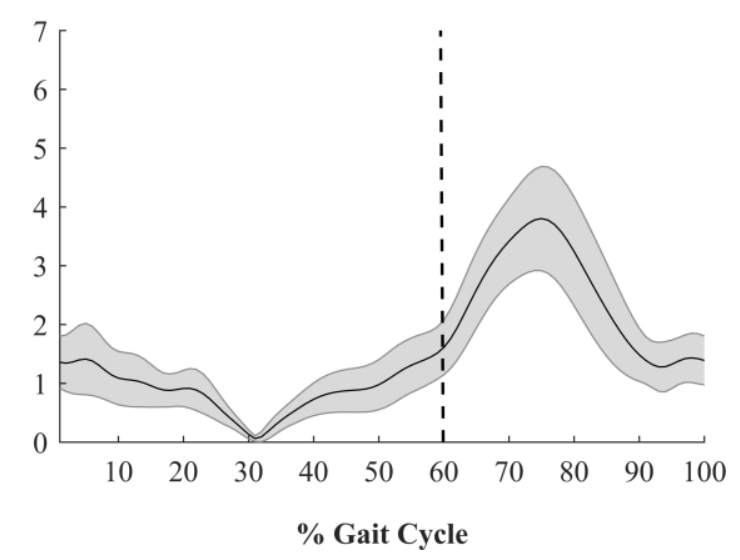

Figure 3: Forefoot, hindfoot, and tibia marker cluster rigid body error $\left(\sigma_{R B E}\right)$ over one full gait cycle calculated with the cluster configurations at mid-stance (50\% stance) as the reference. Means \pm 1 standard deviation $(\mathrm{mm})$. The dotted line indicates the separation between the stance phase and swing phase, with an average of $59.8 \%$ for the population tested in this study. Note that, by definition, $\sigma_{R B E}=0$ at the reference configuration. 


\begin{tabular}{llcccccccc}
\hline & & \multicolumn{2}{c}{ Forefoot marker cluster } & & \multicolumn{2}{c}{ Hindfoot marker cluster } & & \multicolumn{2}{c}{ Tibia marker cluster } \\
& $\mathrm{n}$ & $\sigma_{\text {ave }}$ & $\sigma_{p t p}$ & & $\sigma_{\text {ave }}$ & $\sigma_{\text {ptp }}$ & & $\sigma_{\text {ave }}$ & $\sigma_{p t p}$ \\
\hline Male & 32 & $2.43(0.61)$ & $3.44(1.12)$ & & $1.20(0.55)$ & $0.90(0.29)$ & & $2.287(0.50)$ & $3.64(0.84)$ \\
Female & 32 & $2.33(0.52)$ & $3.24(0.70)$ & & $1.76(1.89)$ & $1.01(0.33)$ & & $2.53(0.51)$ & $3.75(0.73)$ \\
\hline Total & 64 & $2.37(0.57)$ & $3.34(0.94)$ & & $1.47(1.40)$ & $0.96(0.32)$ & & $2.40(0.52)$ & $3.69(0.78)$ \\
\hline
\end{tabular}

Table 1: Average rigid-body error $\left(\sigma_{a v}\right)$ and peak-to-peak rigid-body error $\left(\sigma_{p t p}\right)$ [shown as mean (standard deviation)] for the forefoot, hindfoot, and tibia marker clusters over one full gait cycle, with the static trial used as the reference configuration. Units of $\sigma$ are mm.

\begin{tabular}{|c|c|c|c|c|c|c|c|}
\hline & \multirow[b]{2}{*}{$\mathrm{n}$} & \multicolumn{2}{|c|}{ Forefoot marker cluster } & \multicolumn{2}{|c|}{ Hindfoot marker cluster } & \multicolumn{2}{|c|}{ Tibia marker cluster } \\
\hline & & $\sigma_{a v}$ & $\sigma_{p t p}$ & $\sigma_{a v}$ & $\sigma_{p t p}$ & $\sigma_{a v}$ & $\sigma_{p t p}$ \\
\hline Male & 32 & $2.24(0.50)$ & $4.11(0.95)$ & $0.68(0.15)$ & $1.21(0.29)$ & $1.50(0.24)$ & $3.52(0.69)$ \\
\hline Female & 32 & $2.21(0.34)$ & $4.14(0.67)$ & $0.68(0.21)$ & $1.26(0.37)$ & $1.53(0.24)$ & $3.87(0.85)$ \\
\hline Total & 64 & $2.22(0.43)$ & $4.12(0.82)$ & $0.68(0.18)$ & $1.24(0.33)$ & $1.51(0.24)$ & $3.67(0.79)$ \\
\hline
\end{tabular}

Table 2: Average rigid-body error $\left(\sigma_{a v}\right)$ and peak-to-peak rigid-body error $\left(\sigma_{p t p}\right)$ [shown as mean (standard deviation)] for the forefoot, hindfoot, and tibia marker clusters over one full gait cycle with the cluster configurations at mid-stance (50\% stance) as the reference. Units of $\sigma$ are $\mathrm{mm}$. 


\section{Appendix A}

To assess the repeatability of the measurement protocol for different levels of expertise, as well as the variability of the $\sigma_{R B E}$, one normal male participant underwent eight gait analysis sessions with data collected as described in Section 2.1. Sessions were conducted by two staff members: one (senior) who had 16 years of experience in clinical gait analysis, and another (junior) who had one year of gait analysis and marker-placement experience and no clinical experience. Each staff member performed two sessions on the same day, two hours apart, with the process repeated two weeks later. All markers were removed and reapplied for each new session. The repeatability of $\sigma_{R B E}$ (for the mid-stance reference pose only), defined as the variation in repeated measurements made on the same subject under identical conditions (Bartlett and Frost, 2008), was analyzed using the intertrial error (subject variability), intra-rater error, and inter-rater error (Schwartz et al., 2004). The ratio of the inter-rater error to the inter-trial error was also calculated since it reveals the effect of methodological errors.

Of the three marker clusters considered by the two assessors, the hindfoot cluster had the most repeatable $\sigma_{R B E}$, with the lowest intra- and inter-rater errors (Table A.1). The least reliable $\sigma_{R B E}$ was that for the forefoot cluster, with the highest inter-rater error and interrater/trial ratio. The rater with more experience in gait analysis achieved a more consistent result, as indicated by their lower intra-rater error for all three clusters. The inter-rater to inter-trial ratio was always greater than one. 


\begin{tabular}{|c|c|c|c|c|c|c|c|}
\hline & \multicolumn{3}{|c|}{$\begin{array}{l}\text { Inter-trial error } \\
(\mathrm{mm})\end{array}$} & \multicolumn{2}{|c|}{$\begin{array}{l}\text { Intra-rater error } \\
(\mathrm{mm})\end{array}$} & \multirow{2}{*}{$\begin{array}{l}\begin{array}{l}\text { Inter-rater } \\
\text { error }(\mathrm{mm})\end{array} \\
\text { Mean }\end{array}$} & \multirow[t]{2}{*}{$\frac{\text { Inter-rater }}{\text { Inter-trial }}$} \\
\hline $\begin{array}{l}\text { Marker } \\
\text { cluster }\end{array}$ & Junior & Senior & Mean & Junior & Senior & & \\
\hline Forefoot & 0.157 & 0.130 & 0.143 & 0.230 & 0.154 & 0.257 & 1.79 \\
\hline Hindfoot & 0.080 & 0.075 & 0.077 & 0.105 & 0.094 & 0.110 & 1.42 \\
\hline Tibia & 0.178 & 0.154 & 0.166 & 0.299 & 0.245 & 0.238 & 1.43 \\
\hline
\end{tabular}

Table A.1: Repeatability of $\sigma_{R B E}$, as quantified by the inter-trial, intra-rater and inter-rater errors and the inter-rater/trial ratio. 